
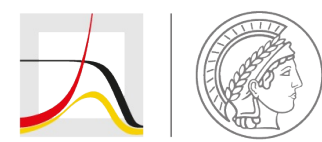

MAX PLANCK INSTITUTE

FOR DEMOGRAPHIC RESEARCH

Konrad-Zuse-Strasse 1 · D-18057 Rostock · Germany · Tel +49 (0) 3812081 - 0 · Fax +49 (0) 3812081 - $202 \cdot$ www.demogr.mpg.de

MPIDR Working Paper WP 2021-001 I February 2021

https://doi.org/10.4054/MPIDR-WP-2021-001

\title{
Mortality change and its impact on child survival
}

\author{
Iván Williams \\ Diego Alburez-Gutierrez I alburezgutierrez@demogr.mpg.de
}

This working paper has been approved for release by: Emilio Zagheni (sekzagheni@demogr.mpg.de),

Head of the Laboratory of Digital and Computational Demography.

(c) Copyright is held by the authors.

Working papers of the Max Planck Institute for Demographic Research receive only limited review. Views or opinions expressed in working papers are attributable to the authors and do not necessarily reflect those of the Institute. 


\title{
Mortality change and its impact on child survival
}

\author{
Iván Williams ${ }^{1}$ and Diego Alburez-Gutierrez ${ }^{2}$ \\ ${ }^{1}$ University of Buenos Aires, Argentina \\ ${ }^{2}$ Lab of Digital and Computational Demography, \\ Max Planck Institute for Demographic Research, Germany
}

February 3, 2021

\begin{abstract}
Background The Goodman-Keyfitz-Pullum Kinship Equations estimate offspring survival for women in populations with stable levels of mortality and fertility. A separate body of literature has focused on the effects of mortality change on life expectancy. Combining these two approaches allows us to consider the effects of mortality change on offspring survival.

Results The effect of an all-age mortality increase on child survival is indirectly proportional to the mean age of an average woman's surviving children. Equivalently, the effect is indirectly proportional to the mean time that a woman has spent with her surviving offspring. Generalizing for an age-specific mortality change follows life expectancy treatment.

Contributions We bring together two separate traditions in mathematical demography to provide an intuitive understanding of the consequences of mortality change for offspring survival and women's lived experience of child loss. This conceptual framework can be used, for example, to study maternal bereavement in the context of a sustained mortality decline or a sudden mortality crisis.
\end{abstract}

\section{Relationship}

Let $C S_{a}=\int_{0}^{a} m_{x} l_{a-x} d x$ be the expected number of children surviving to a woman aged $a$ in a female stable population with fertility rates $m_{x}$, mortality hazard $\mu_{x}$, and survival function $l_{x}=e^{-\int_{0}^{x} \mu_{t} d t}$ (with unit radix $l_{0}=1$ ), as described by Goodman, Keyfitz, \& Pullum (1974). The effect of an absolute change $\Delta \delta$ (positive or negative) in mortality rates (resulting in $\mu^{*}=\mu+\delta$ ), on child survival $C S_{a}$ for a woman aged $a$, is inversely proportional to the mean age of her surviving daughters $\bar{x}_{a}$ : 


$$
\frac{\Delta C S_{a}^{*}}{C S_{a}} \approx-\bar{x}_{a} \Delta \delta
$$

This change applies only to surviving offspring, like an excess mortality effect, and the magnitude of the change is more pronounced the younger the fertility profile is. Equation 1, in which mortality increases uniformly at all ages, is a special case of a more general formal relation linking age-specific changes in mortality to child survival from a mother's perspective.

\section{Proof}

The average number of surviving daughters for a woman aged $a$ can be thought of in two ways: $C S_{a}=$ $\int_{0}^{a} m_{x} l_{a-x} d x=\int_{0}^{a} m_{a-x} l_{x} d x$, depending on whether $x$ represents the mother's or the daughter's age. The latter expression is akin to the temporary life expectancy ${ }_{a} e_{0}$, but with fertility $m$ acting as a cohort size factor in a stationary context (i.e., a special kind of Markov 'rewards' (Caswell, 2019)). Given this similitude, as a first step we can generalize the Wrycza \& Baudisch (2012) approach for changing rates $\mu_{x}^{*}$ in the age range $(b, c)$ with $0 \leq b \leq c \leq a$ and $c=b+h$ to see its impact on ${ }_{a} e_{0}$ :

$$
{ }_{a} e_{0}^{*}=\int_{0}^{a} e^{\left[-\int_{0}^{\min (b, x)} \mu_{t} d t-\int_{\min (b, x)}^{\min (c, x)} \mu_{t}^{*} d t-\int_{\min (c, x)}^{a} \mu_{t} d t\right]} d x
$$

The expression min returns the minimum between two numbers. Replacing $\mu_{t}^{*}=\mu_{t}+\delta$ and rearranging the terms corresponding to $l_{x}$, for a small absolute change $(\delta \rightarrow 0)$ we get:

$$
\begin{gathered}
{ }_{a} e_{0}^{*}=\int_{0}^{a} l_{x} e^{-[\min (c, x)-\min (b, x)] \delta} d x \\
\frac{d_{a} e_{0}^{*}}{d \delta} \approx-\int_{0}^{a} l_{x}[\min (c, x)-\min (b, x)] d x \\
\frac{d_{a} e_{0}^{*}}{d \delta} \approx-\left[\int_{0}^{c} l_{x} x d x-\int_{0}^{b} l_{x} x d x+\int_{c}^{a} l_{x} c d x-\int_{b}^{a} l_{x} b d x\right] \\
\approx-\left[\int_{b}^{c} l_{x}(x-b) d x+\int_{c}^{a} l_{x} h d x\right]
\end{gathered}
$$




$$
\begin{aligned}
\frac{d_{a} e_{0}^{*}}{d \delta} & \approx-\left[\left(T_{b}-T_{c}\right)\left(\bar{x}_{b, c}-b\right)+\left(T_{c}-T_{a}\right) h\right] \\
& \approx-\left[C_{b, c}\left(\bar{x}_{b, c}-b\right)+C_{c, a} h\right] e_{0} .
\end{aligned}
$$

$T_{b}$ is the population over some age $b$ and $C_{b, c}$ is its weight in the total population; in this case, between ages $b$ and $c$. This derivative depends on life expectancy $e_{0}$ (i.e., its default level) and a population-weighted measure of expected time for women aged between b and c (first term), and women older than c (second term). When $a=c=\omega$ and $b=0$ this expression is $\bar{x} e_{0}$ : the mean age in a stationary population times life expectancy at birth (Wrycza \& Baudisch, 2012).

Returning to child survival $C S_{a}$, we now include the fertility factor in Equation 4:

$$
\frac{d C S_{a}^{*}}{d \delta} \approx-\left[\int_{b}^{c} m_{a-x} l_{x}(x-b) d x+h \int_{c}^{a} m_{a-x} l_{x} d x\right]
$$

The relative impact of an absolute change in mortality within ages b and c on child survival is:

$$
\frac{\Delta C S_{a}^{*}}{C S_{a}} \approx-\left[\frac{C S_{a}^{b, c}}{C S_{a}}\left(\bar{x}_{a}^{b, c}-b\right)+\frac{C S_{a}^{c, a}}{C S_{a}} h\right] \Delta \delta .
$$

$C S_{a}^{b, c}$ represents the expected number of surviving daughters aged between $b$ and $c$ for a mother aged $a$, and $\bar{x}_{a}^{b, c}$ represents their mean age. Equations 5 and 7 share the same intuition, but the latter is weighted by daughter survival within each age range at maternal age $a$. To get Equation 1 in the Relationship section, consider a change over the entire age range $(a=c<\omega$ and $b=0)$. The relative impact of an absolute change in mortality rates on $C S_{a}$ is proportional to the mean age of a woman's surviving daughters $\bar{x}_{a}$ :

$$
\frac{\Delta C S_{a}^{*}}{C S_{a}} \approx-\bar{x}_{a} \Delta \delta
$$

Finally, we prove that an equivalent expression can be derived in terms of the mother's lived years. Let $x$ be the mother's age and $a=c<\omega$ and $b=0$, then:

$$
C S_{a}^{*}=\int_{0}^{a} m_{x} l_{a-x} e^{-\delta(a-x)} d x
$$




$$
\begin{aligned}
\frac{d C S_{a}^{*}}{d \delta} & \approx-a \int_{0}^{a} m_{x} l_{a-x} d x+\int_{0}^{a} x m_{x} l_{a-x} d x \\
& \approx-a C S_{a}+\int_{0}^{a} x m_{x} l_{a-x} d x
\end{aligned}
$$

Dividing both sides by $C S_{a}$ we get the discrete approximation:

$$
\frac{\Delta C S_{a}^{*}}{C S_{a}} \approx-\left(a-k_{a}\right) \Delta \delta
$$

Equation 11 shows that the expected change in offspring survival is inversely proportional to the difference between maternal age $a$ and the mean age of the mother at the birth of her surviving daughters $k_{a}$. By intuition, this is equivalent to the mean age of the mother's surviving daughters, as shown in Equation 1.

\section{Related relationship}

\section{An approximation}

We approximate $l_{x}$ using a second-order Taylor polynomial around the mean age of childbearing $\kappa$ to obtain an intuitive understanding of the demographic forces that drive child survival:

$$
\begin{aligned}
C S_{a} & \approx l_{a-\kappa} \int_{0}^{a} m_{x} d x+\left(l_{a-\kappa}\right)^{\prime} \int_{0}^{a}(x-\kappa) m_{x} d x+\left(l_{a-\kappa}\right)^{\prime \prime} \int_{0}^{a} \frac{(x-\kappa)^{2}}{2} m_{x} d x \\
& \approx F_{a} l_{a-\kappa}+\frac{\sigma^{2}}{2} F_{a}\left(l_{a-\kappa}\right)^{\prime \prime} \\
& \approx F_{a} l_{a-\kappa}\left[1+\frac{\sigma^{2}}{2} \frac{\left(l_{a-\kappa}\right)^{\prime \prime}}{l_{a-\kappa}}\right] .
\end{aligned}
$$

Here, the fertility pattern by age is concentrated around $\kappa$, and the accumulated fertility (or the gross reproduction rate in our female-only scenario) is $F_{a}=\int_{\alpha}^{a} m_{x} d x$. The second Taylor's term is null because $\int_{\alpha}^{a} x m_{x} d x=\kappa F_{a}$. We find that, seen from the perspective of a mother, child survival mainly depends on the cumulative fertility function and the survival of daughters from birth to age $a-\kappa$. The approximation is affected negatively by the dispersion of fertility over age (variance $\sigma^{2}$ ) and the (negative) curvature of the survival curve $l_{x}$ in the age range $20-40$, which is typically very flat in post-transition populations ${ }^{1}$.

\footnotetext{
${ }^{1}$ This approximation also highlights the relevance of changes in the mean age of childbearing for child survival if fertility levels remain unchanged. After taking the logs of $F_{a} l_{a-\kappa}$ and deriving we get $\frac{\Delta C S_{a}}{C S_{a}} \approx \mu_{a-\kappa} \Delta \kappa$. This shows that daughters are exposed to a shorter mortality risk period because of a hypothetical fertility delay.
} 


\section{Burden of maternal bereavement}

The Shared-Lifetime Lost (SLT) is a measure of the daughter person-years 'lost' to a mother because of the death of her daughter. It is also the expected time that a mother aged $a$ could have spent with a living daughter if each daughter would have survived at each age. This can be expressed in terms of a temporary expected lost years index, in line with $e^{\dagger}$ (Vaupel, 1986):

$$
S L T_{a}=\int_{0}^{a} m_{a-x} \int_{0}^{x} d_{t[x-t]} e_{0} d t d x=\int_{0}^{a} m_{[a-x] x} e_{0}^{\dagger} d x
$$

where $d_{t}$ is the death distribution from birth, ${ }_{[x-t]} e_{0}$ is the life expectancy at birth until age $x-t$, and ${ }_{x} e_{0}^{\dagger}$ is the temporary dispersion measure.

A related measure compares these 'lost' years to the years that a mother could have shared with her daughters. We call this the Intensity of Time Lost (ITL): a ratio between the expected time spent bereaved (in daughter person-years lost, as introduced above) and the expected time spent with living daughters:

$$
\operatorname{ITL} L_{a}=\frac{\int_{0}^{a} m_{[a-x] x} e_{0}^{\dagger} d x}{\int_{0}^{a} m_{[a-x] x} e_{0} d x} .
$$

This measure allows us to compare these 'lost' years across different population regimes. It is also similar to the transcendental entropy measure $\bar{H}$ (Keyfitz \& Caswell, 2005), but considering all the cohorts born during the mother's life, weighted by their relative size. Assuming that fertility is concentrated around $\kappa$ (see An approximation), daughters would have $a-\kappa$ years of exposure to death, on average. Thus, we can express this measure as $I T L_{a} \approx \bar{H}_{a-\kappa}$, the entropy measure restricted to $a-\kappa$. A woman's intensity of loss is proportional to the lifespan inequality of her daughters.

\section{Applications}

Using data from countries in Latin America and the Caribbean, we consider the impact of an absolute change of mortality of .001 on the expected number of living daughters for an average woman aged 50 whose surviving daughters are, on average, 25 years old. We chose this region as an example because it includes countries with widely differing levels of fertility and mortality. If mortality in the region increased uniformly across age ${ }^{2}$, the number of living daughters would decrease by $2.5 \%\left(\frac{\Delta C S_{50}}{C S_{50}} \approx 25 * 0.001\right)$ (Equation 1$)$. What would happen if mortality increased only between ages 15-29, and remained constant at all other ages? Assuming that 80\%

\footnotetext{
${ }^{2}$ For reference, mortality rates in Latin America and the Caribbean in 1950-1955 were 0.1255, 0.0174, 0.0037, 0.0019, 0.0029, 0.0041 , and 0.005 for, respectively, ages zero, 1-4, and then grouped by five until age 25; which implies a different relative impact for each group.
} 
of the woman's daughters are aged 15-29, this change would decrease the woman's parity by approximately $\left[.8^{*}(25-15)+.2 * 15\right]^{*} .001,1.1 \%$ (Equation 7$)$.

This framework has many practical applications. For example, it can be used to approximate the effect of a mortality crisis on maternal bereavement. Using traditional demographic data from censuses and household surveys, it can reveal inequalities in the experience of maternal bereavement by socioeconomic status (SES). How much would mortality need to decline for lower-SES women to experience the same levels of child survival as higher-SES women, controlling for fertility levels (i.e., $\left.\Delta \delta \approx\left[C S_{a}^{\text {lower }}-C S_{a}^{\text {higher }}\right] /\left[\bar{x}_{a}^{\text {lower }} C S_{a}^{\text {lower }}\right]\right)$ ?

Figure 1 shows the effect of a mortality change $\Delta \delta$ on child survival $C S_{a}$ at different maternal ages based on two alternative estimation methods. 'Empirical' estimates come from a discretized form of the equation $C S_{a}=\int_{0}^{a} m_{x} l_{a-x} d x$, and 'approximated' values come from Equation $1^{3}$. The figure shows that the effect of $\Delta \delta$ is larger at older maternal ages. We find that the estimates from the empirical and approximated methods are broadly consistent, especially for small $\Delta \delta$. However, our approximation tends to overestimate the effect before maternal age 40, and to underestimate it after that age when the change is large.

We now evaluate the accuracy of Equation 12, an approximation of $C S_{a}$ introduced in the Related relationship section to capture essential factors of its behaviour by age. Figure 2 (panels A-C) shows that this approximation is very precise, especially for recent periods. The relative error $\left(\frac{\text { aprox-empirical }}{\text { empirical }}\right)$ across all regions and years for $a=30$ ranges from $.8 \%$ to $.005 \%$, and from $-.8 \%$ to $-.08 \%$ for age 50 . The accuracy of the approximation increased over time, as exemplified by Guatemala (red dots in Figure 2). Panels D-E show that for the Guatemalan case, this improvement can be explained by the rectangularization of $l_{x}$ (as lower mortality at young ages leads to second derivatives close to zero in that range), and, to a lesser degree, by lower dispersion in the fertility age pattern over time.

Lastly, we consider the Intensity of Time Lost (ITL), introduced in the Burden of maternal bereavement section. Figure 3 shows the ratio between the daughter person-years 'lost' by an average woman and the total daughter person-years that she would have shared with her daughters in the absence of offspring death (Equation 14). Higher values indicate a more 'intense' experience of bereavement up to maternal age $a$. The ITL for Haitian women at age 30 is $4.8 \%$ for those born in 1950-1955 and is $1.4 \%$ for those born in 2010-2015. The equivalent values for Costa Rican women are $2.3 \%$ and $0.2 \%$. The average values in the region are $2.7 \%$ and $.3 \%$ for the most recent period. Offspring survival at higher maternal ages depends less on infant mortality, which is why ITL values are lower than $2 \%$ for women aged 50 in all countries, eventually

\footnotetext{
${ }^{3}$ Using fertility and mortality rates from the 2019 Revision of the United Nations World Population Prospects. We smoothed female $l_{x}$ using cubic-splines constrained to monotonic decrease, taking $L_{0}$ and $T_{100}$ from raw life tables as inputs for person-years calculations. We obtained single-year fertility rates using a quadratic optimization approach (Michalski et al., 2018). We assumed a sex ratio at birth of 0.49 for all periods and countries. The discrete calculations assumed that live births $m_{x}$ happen at exact maternal age $x$. All reproducible materials are available in the online Appendix.
} 


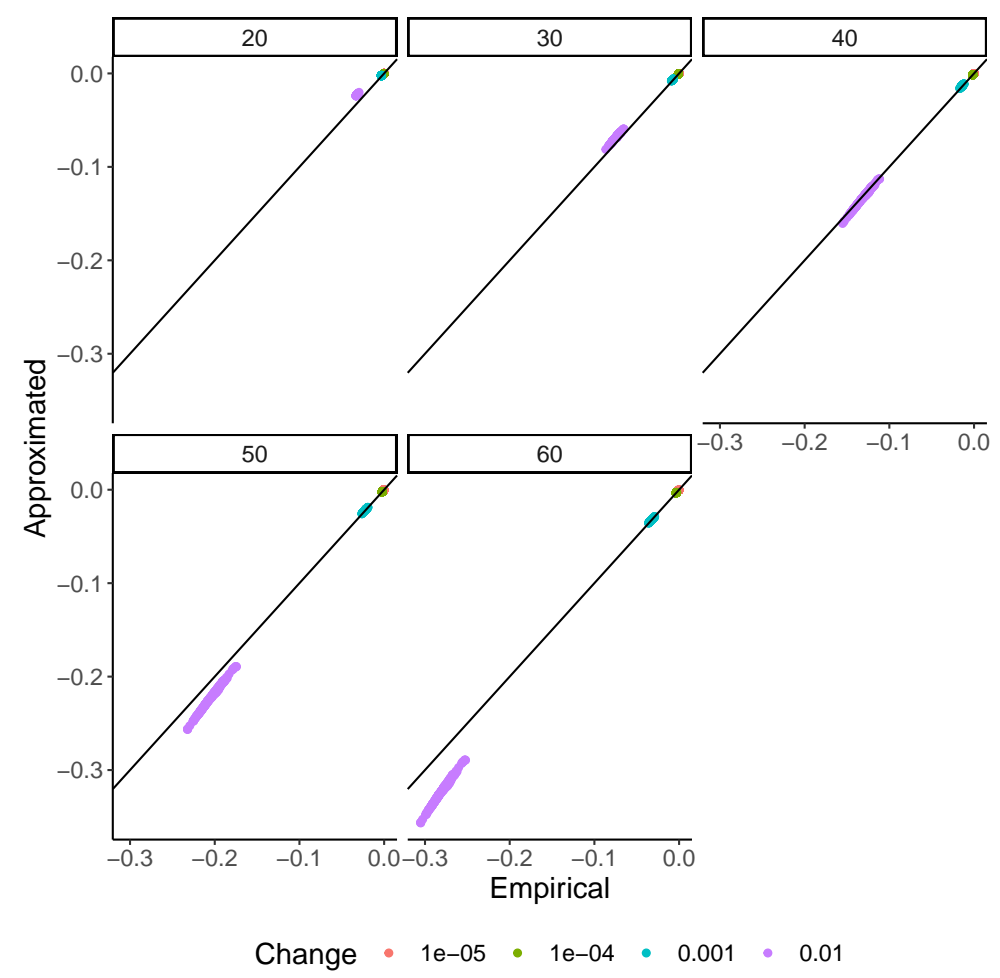

Figure 1: Estimate and empirical $C S_{a}$ for different mother ages and change sizes. Latin America countries in the 1950-2015 period

converging to zero.

In conclusion, our work contributes to the formal understanding of the effects of mortality change, age-specific or uniform over age, on the experience of offspring survival and offspring loss for women or, with some modifications, for men. We identify two main limitations of our approach. First, it assumes a homogeneous population with stable fertility and mortality, and it assumes that mortality change is permanent and affects all birth cohorts. Second, a uniform and absolute mortality change over a wide age interval (e.g., ages 0-40) may be unrealistic. One way of addressing this issue would be to account for other types of mortality change (proportional, exponential, lineal, etc.) (Wrycza \& Baudisch, 2012). Alternatively, Equations 2-7 could be used to concatenate a series of age-specific (absolute) mortality changes. These interesting lines of work lie outside the scope of this paper, but we hope that our approach will inspire the further development of indirect estimations methods applicable to widely available demographic data.

\section{History}

Kin count estimation has a long history in mathematical demography, starting with the work of Lotka (1931) on modelling orphanhood in theoretical populations across demographic regimes. To the best of our 

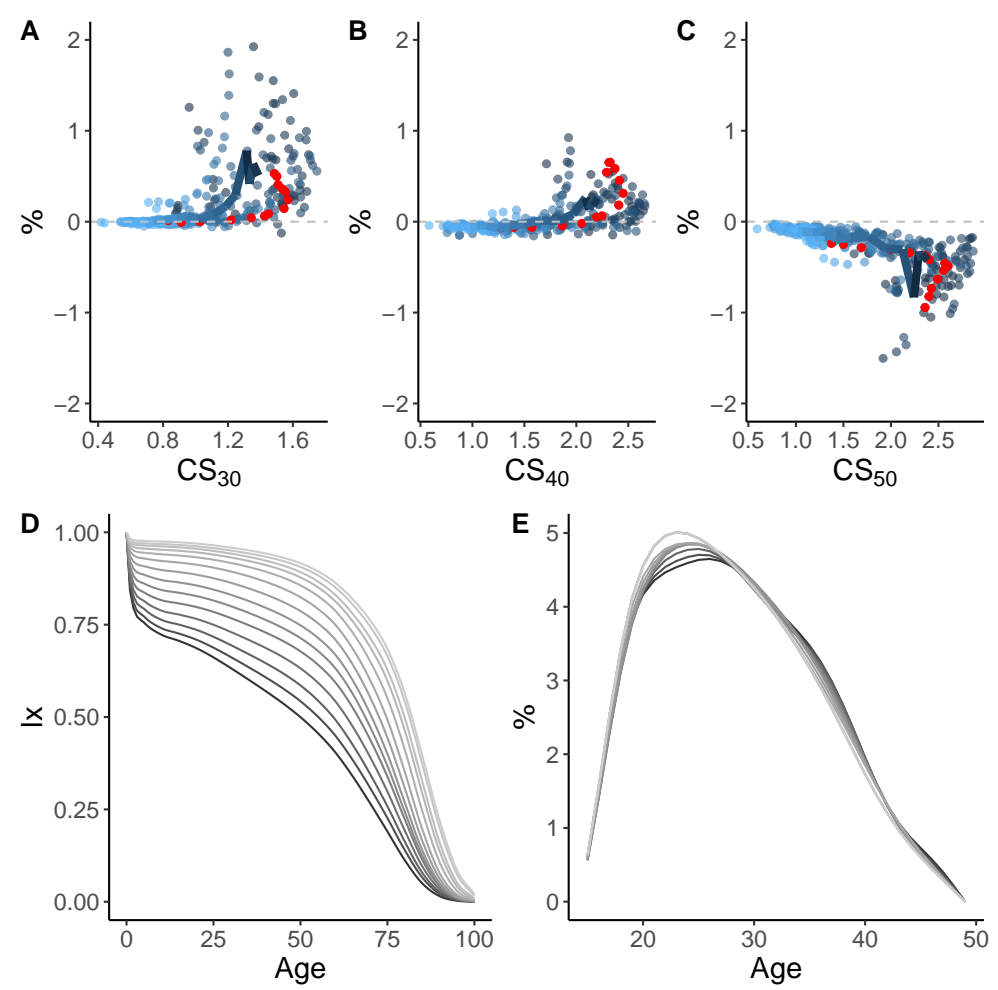

Figure 2: Relative error in approximation of child survival $C S_{a}$ for ages 30 (A), 40 (B), and 50 (C), from the years 1950-1955 (darker) to the years 2015-2020 (lighter) for all Latin American countries (blue) and Guatemala (red). D) Guatemala: $l_{x}$, same period. E) Fertility distribution by age in Guatemala, same period.

knowledge, Brass (1953) first proposed an equation akin to $C S_{a}=\int_{0}^{a} m_{x} l_{a-x} d x$ to estimate child survival over maternal age. Goodman et al. (1974) generalized this approach to sisters, granddaughters, cousins, etc., in stable and non-stable populations. The so-called Goodman-Keyfitz-Pullum Kinship Equations were popularized by Keyfitz \& Caswell (2005), even though Bongaarts (1987) used a similar approach to estimate descendants in his 'Family Status Model'. More recently, Caswell (2019) recast the problem of kin counts using matrix algebra. Finally, Wrycza \& Baudisch (2012) used formal techniques to describe the effect of different types of age-specific mortality change on life expectancy, an approach we drew upon heavily in this paper.

\section{Acknowledgments}

We thank Tim Riffe for useful feedback. The paper was discussed at the Work-in-progress Workshop of the MPIDR Lab of Digital and Computational Demography. 


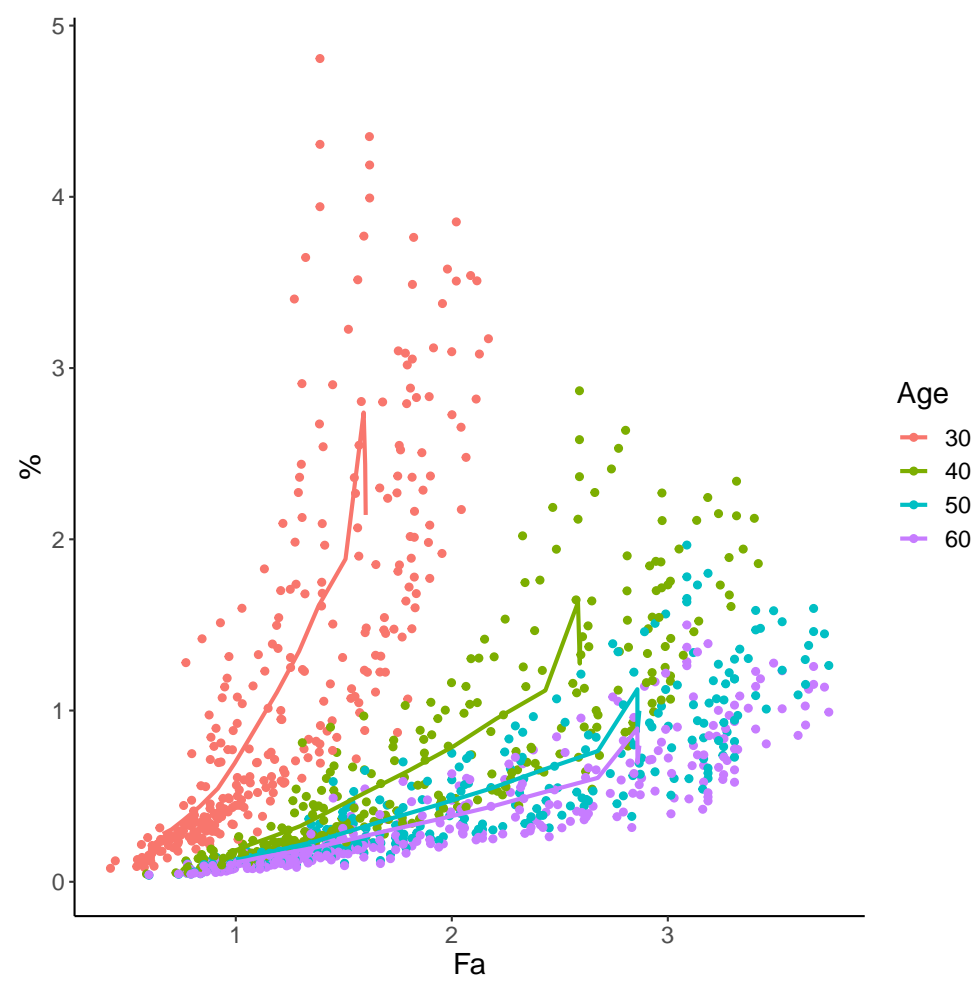

Figure 3: Intensity of Time Lost (ITL) by fertility levels and maternal age. All Latin American countries in the 1950-2015 period, estimated using data from the 2019 United Nations World Population Prospects.

\section{References}

Bongaarts, J. (1987). The Projection of Family Composition over the Life Course with Family Status Life

Tables. In J. Bongaarts \& T. Burch (Eds.), Family Demography: Methods and their Application. Oxford: IUSSP Series, Oxford Univer- sity Pres.

Brass, W. (1953, November). The Derivation of Fertility and Reproduction Rates from Restricted Data on Reproductive Histories. Population Studies, 7(2), 137. Retrieved 2019-08-07, from https://www.jstor .org/stable/2172029? origin=crossref doi: 10.2307/2172029

Caswell, H. (2019, September). The formal demography of kinship: A matrix formulation. Demographic Research, 41, 679-712. Retrieved 2019-09-17, from https://www.demographic-research.org/volumes/ vol41/24/ doi: 10.4054/DemRes.2019.41.24

Goodman, L. A., Keyfitz, N., \& Pullum, T. W. (1974). Family formation and the frequency of various kinship relationships. Theoretical Population Biology, 5(1), 1-27.

Keyfitz, N., \& Caswell, H. (2005). Applied mathematical demography (Vol. 47). Springer.

Lotka, A. J. (1931). Orphanhood in relation to demographic factors. Metron, 9(2), 37-109. 
Michalski, G. P., Anatoli I, , \& Gorlishchev, V. P. (2018). $R$ programs for splitting abridged fertility data into a fine grid of ages using the quadratic optimization method (Tech. Rep.). Max Planck Institute for Demographic Research, Rostock, Germany.

Vaupel, J. W. (1986, Mar). How Change in Age-Specific Mortality Affects Life Expectancy. Population Studies, 40(1), 147-157. doi: 10.2307/2174285

Wrycza, T., \& Baudisch, A. (2012). How life expectancy varies with perturbations in age-specific mortality. Demographic Research, 27, 365-376. 